\title{
Phase Field Simulation for Grains Evolution of 17-4PH Steel During Cyclic Heat Treatment
}

\author{
Yuan YOU, Mufu YAN $N^{\dagger}$ and Chengsong ZHANG
}

National Key Laboratory for Precision Hot Processing of Metals, School of Materials Science and Engineering, Harbin Institute of Technology, Harbin 150001, China

[Manuscript received 24 March 2012, in revised form 28 July 2012]

(c) The Chinese Society for Metals and Springer-Verlag Berlin Heidelberg

\begin{abstract}
A phase field model is developed to simulate the grain evolution of $17-4 \mathrm{PH}$ steel during cyclic heat treatment (CHT). Our simulations successfully reproduce the grain morphologies of every CHT. In the process of every $\mathrm{CHT}$, phase transformation recrystallization happens. The recrystallized grains appear mainly on the original grain boundaries. The average grain size of $13.2 \mu \mathrm{m}$ obtained by $1040{ }^{\circ} \mathrm{C} \times 1 \mathrm{~h}$ solution treatment for this experimental steel can be refined to $2.2 \mu \mathrm{m}$ after five $\mathrm{CHT}$ 's. Furthermore, the effects of phenomenological parameters in our model are discussed.
\end{abstract}

KEY WORDS: Phase field model; Grain refinement; Recrystallization; 17-4PH Steel

\section{Introduction}

17-4PH steel has a variety of applications because of its high strength and excellent corrosion resistance. In order to extend the range of its applications, we carry out cyclic heat treatment (CHT) to refine its grains. According to Hall-Petch equation, grain refinement is an effective way to improve the strength of materials. After CHT, the grains of the steel can be refined, this is because phase transformation recrystallization happens. Although the microstructure of refined grains can be observed by optical microscope, the process of the grains evolution remains unclear. Computer simulation is an appropriate tool to explore the process of recrystallization.

Several techniques for the computer simulation of recrystallization have been developed in the last decade, including Monte Carlo models ${ }^{[1-4]}$, cellular automata $^{[5-8]}$, molecular dynamics ${ }^{[9,10]}$. The phase field method has its own great advantages for the simulations of the microstructure evolution of materials during recrystallization. It allows not only to monitor

\footnotetext{
† Corresponding author. Prof., Ph.D.; Tel: +86 45186418617 ; Fax: +86 451 86413922; E-mail address: yanmufu@hit.edu.cn (Mnfu YAN)
}

DOI: $10.1007 / \mathrm{s} 40195-012-0505-6$ the microstructure evolution during recrystallization but also to obtain the useful information about the average grains size and the size distribution. Recently, phase-field models were employed to simulate dendritic growth ${ }^{[11-13]}$. Suwa et al. ${ }^{[14,15]}$ developed a phase field method coupling the unified subgrain growth theory to investigate the effects of the microstructural inhomogeneities on the recrystallization kinetics. Wang et al. ${ }^{[16]}$ established a phase-field model to simulate the realistic spatio-temporal microstructure evolution in recrystallization of a magnesium alloy. Takaki et al. ${ }^{[17]}$ developed a multi-phasefield model to simulate dynamic recrystallization during grain deformation. It should be emphasized that all of these methods are limited to deformation recrystallization, while the study on the phase transformation recrystallization is seldom reported.

In this study, we attempt to develop a phase field model to simulate the grain refinement of $17-4 \mathrm{PH}$ steel during cyclic heat treatment, which belongs to phase transformation recrystallization occurring during the processes from martensite to austenite transformation. Furthermore, we reveal the effects of the parameters in the present phase field model on the evolution of the grain morphologies and discuss the relations between the phase field parameters and the experimental parameters. 


\section{Material and Methods}

\subsection{Material and treatments}

The as-received $17-4 \mathrm{PH}$ steel with the chemical composition (wt.\%) 0.06 C, $17.22 \mathrm{Cr}, 3.91 \mathrm{Ni}, 3.01$ $\mathrm{Cu}, 0.16 \mathrm{Nb}, 0.81 \mathrm{Si}, 0.76 \mathrm{Mo}$, and balance Fe was used. The steel bar was solution treated (ST) at $1040{ }^{\circ} \mathrm{C}$ for $1 \mathrm{~h}$ and then cooled in water. The CHT samples with a dimension of $10 \mathrm{~mm} \times 10 \mathrm{~mm} \times 6 \mathrm{~mm}$ are heated at $885{ }^{\circ} \mathrm{C}$ for $10 \mathrm{~min}$ in salt-bath furnace and then quenched in water. The $\mathrm{CHT}$ was repeated from 1 to 5 times.

The cross-sectioned samples after CHT were ground and polished, and then etched in a mixed solution of $1 \mathrm{~g} \mathrm{KMnO} 4+10 \mathrm{~mL} \mathrm{H}_{2} \mathrm{SO}_{4}+100 \mathrm{~mL} \mathrm{H}_{2} \mathrm{O}$. The grain morphology of the etched samples was observed on an optical microscope.

\subsection{Phase field model}

In our simulated system, there exist two kinds of grains, i.e. the original grains and the recrystallized ones. Two sets of field variables $\left(\eta_{\mathrm{a} 1}, \eta_{\mathrm{a} 2}\right.$, $\left.\cdots, \eta_{\mathrm{a} m}\right)$ and $\left(\eta_{\mathrm{b} 1}, \eta_{\mathrm{b} 2}, \cdots, \eta_{\mathrm{b} n}\right)$ are employed to describe various orientations of the original and recrystallized grains, respectively, in which a corresponds to the original grains, b corresponds to the recrystallized grains, $m$ and $n$ represent the number of orientation field variables.

According to the diffuse-interface theories ${ }^{[18]}$, the total energy, $F$, of the system can be approximated as follows:

$$
\begin{gathered}
F=\int\left\{f_{0}\left[\left\{\eta_{i}(r)\right\}\right]+\sum_{i} \frac{\kappa_{i}}{2}\left[\nabla \eta_{i}(r)\right]^{2}\right\} \mathrm{d}^{3} r, \\
i=\mathrm{a} 1, \mathrm{a} 2, \cdots, \mathrm{a} m, \mathrm{~b} 1, \mathrm{~b} 2, \cdots, \mathrm{b} n
\end{gathered}
$$

where $f_{0}$ is the local energy density, which is a function of all the field variables, $\nabla \eta_{i}$ are the gradient of field variables, $\kappa_{i}$ are the corresponding gradient energy coefficients.

According to the Ref. [19-21], we assume the local energy density function $f_{0}$ in the present system as follows:

$$
\begin{aligned}
& f_{0}\left(\left\{\eta_{i}\right\}\right)=\sum_{i}\left[\beta\left(-\frac{\alpha^{2}}{2} \eta_{i}^{2}+\frac{1}{4} \eta_{i}^{4}\right)\right]+\gamma \sum_{i} \sum_{j>i} \eta_{i}^{2} \eta_{j}^{2}, \\
& i, j \in\{\mathrm{a} 1, \mathrm{a} 2, \cdots, \mathrm{a} m, \mathrm{~b} 1, \mathrm{~b} 2, \cdots, \mathrm{b} n\} \text { and } j>i
\end{aligned}
$$

where $\alpha, \beta$ and $\gamma$ are phenomenological parameters. Every term in the first summation in the right side of Eq. (2) is a double-well potential with the wells located at $\eta_{i}=\alpha$ or $\eta_{i}=-\alpha$. The cross terms are added to ensure that the local energy density function has $2(m+n)$ degenerate minima.

Actually, the grain evolution in the present model depends on the double-well potential in Eq. (2). We denote $f$ as follows:

$$
f=-\frac{\alpha^{2}}{2} \eta^{2}+\frac{1}{4} \eta^{4}
$$

When the temperature of the system rises, the total energy of the system will increase. Therefore, we assume that phenomenological parameter $\alpha$ is a function of temperature, i.e. $\alpha=\alpha(T)$. The curves of the local energy density $(f)$ as a function of one orientation field variable $(\eta)$ for different parameters of $\alpha$ are shown in Fig. 1. We can see that the local energy density increases with decreasing the values of parameters $\alpha$. Thus the reduction of $\alpha$ value can describe the increase of the system's temperature, i.e. the increase of the total energy of the system. It can also be easily obtained that the two minima of the local energy density locate at $\eta= \pm \alpha$.

The spatial and temporal evolution of the orientation field variables is described by Ginzburg-Landau equations:

$$
\begin{gathered}
\frac{\partial \eta_{i}(r, t)}{\partial t}=-L_{i} \frac{\delta F}{\delta \eta_{i}(r, t)}+\xi_{i}(r, t), \\
i=\mathrm{a} 1, \mathrm{a} 2, \cdots, \mathrm{a} m, \mathrm{~b} 1, \mathrm{~b} 2, \cdots, \mathrm{b} n
\end{gathered}
$$

where $L_{i}$ are the kinetic coefficient relative to grain boundary mobility, $t$ is time, and $\xi_{i}(r, t)$ is noise terms following the distribution of Gaussian. Substituting the Eq. (1) into the Eq. (4) gives:

$$
\begin{gathered}
\frac{\partial \eta_{i}}{\partial t}=-L_{i}\left[\frac{\partial f_{0}\left(\left\{\eta_{i}\right\}\right)}{\partial \eta_{i}}-\kappa_{i} \nabla^{2} \eta_{i}\right], \\
i=\mathrm{a} 1, \mathrm{a} 2, \cdots, \mathrm{a} m, \mathrm{~b} 1, \mathrm{~b} 2, \cdots, \mathrm{b} n
\end{gathered}
$$

Using the local energy density as Eq. (2), we obtain the final set of kinetic equations:

$$
\begin{gathered}
\frac{\partial \eta_{i}}{\partial t}=-L_{i}\left[\beta\left(-\alpha_{i}^{2} \eta_{i}+\eta_{i}^{3}\right)+2 \gamma \eta_{i} \sum_{j \neq i} \eta_{j}^{2}-\kappa_{i} \nabla^{2} \eta_{i}\right] \\
i, j \in\{\mathrm{a} 1, \mathrm{a} 2, \cdots, \mathrm{a} m, \mathrm{~b} 1, \mathrm{~b} 2, \cdots, \mathrm{b} n\} \text { and } j \neq i
\end{gathered}
$$

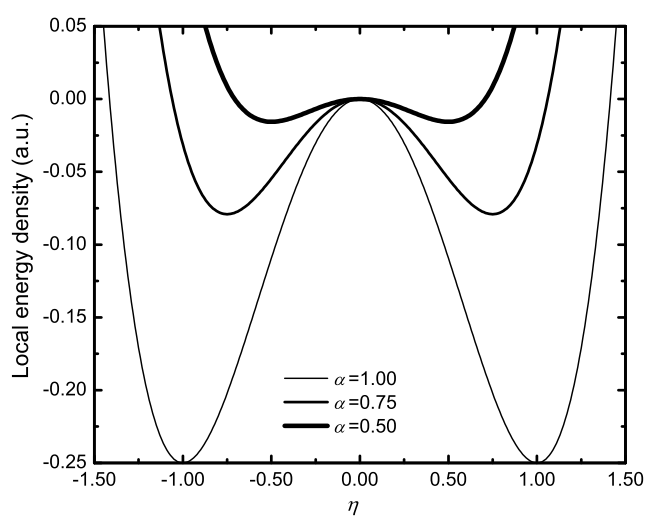

Fig. 1 Local energy density curves with different phenomenological parameters of $\alpha$ 


\section{Results and Discussion}

We employ the parameters in Table 1 and periodic boundary conditions along both Cartesian coordinate axes to simulate numerically the grain evolution of 17 4PH steel during ST and CHT. As well known, above the transformation point the recrystallized grain is more stable than the original one. Therefore, we give the parameter $\alpha_{\mathrm{a}}$ a smaller value (0.76) for the original grains, and give $\alpha_{\mathrm{b}}$ a greater value (1) for the recrystallized grains. The difference of the local energy density caused by the two $\alpha$ values can be observed from Fig. 1. The recrystallized grains possess lower energy and more stable than the original ones. Moreover, the gradient energy coefficient $\left(\kappa_{\mathrm{a}}\right)$ of the original grains is assigned a small value (0.1) to decrease the effects of the original grain boundaries on the formation and growth of the recrystallized grains.

The grains evolution of $17-4 \mathrm{PH}$ steel during cyclic heat treatment is simulated using the present phase field model as follows:

(1) The first step is to simulate the grains after $\mathrm{ST}$ with a set of orientation field variables $(m=30)$. Every orientation field variable owns a value on every grid point in our simulated system. On all the grid points in a certain grain, the square of a certain $\eta_{\mathrm{a} i}$, i.e. $\eta_{\mathrm{a} i}^{2}$, is equal to 1 . In its neighbor grain, the square of another certain $\eta_{\mathrm{a} j}$, i.e. $\eta_{\mathrm{a} j}^{2}$, is equal to 1 . On the grain boundary from this grain to another, the value of $\eta_{\mathrm{a} i}^{2}$ decreases gradually from 1 to 0 , and the value of $\eta_{\mathrm{a} j}^{2}$ increases gradually from 0 to 1 .

(2) After the first CHT, another set of the orientation field variables is added to describe the recrystallized grains $(n=10)$. As initial conditions for this step, the original orientation field variables are remained and the new orientation field variables are assigned small random values between -0.01 and 0.01 . Every orientation field variable for the recrystallized grains owns a small random value on every grid point in our simulated system.

(3) To simulate the grains after two times CHT, we use the grains after the first CHT as the original ones and add again a new set of orientation field variables as initial conditions for this step. The total number of the orientation field variables for this step is 50 , in which there are 30 variables arising from the first step for simulating the grains after ST, 10 variables from the second step for the first $\mathrm{CHT}$, and 10 variables for the second CHT.

(4) Repeating the procedure (3) described above, we can simulate the grains after every CHT.

Fig. 2 presents the experimental (Fig. 2(a)-(f)) and simulated (Fig. 2(g)-(l)) grain morphologies of 17-4PH steel treated by ST and ST+CHT.

As shown in Fig. 2(a) and (g), the grains obtained by $\mathrm{ST}$ are coarse, and the grain boundary is relatively regular. Fig. 2(b)-(f) and Fig. 2(h)-(l) show the grain morphologies after every $\mathrm{CHT}$, respectively. It can be seen from Fig. 2(b) and (h) that the original grains can still be observed. However, there are a lot of small recrystallized grains formed on the original grain boundaries rather than inside the grains. In an original grain, the square of a certain $\eta_{\mathrm{a} i}$, i.e. $\eta_{\mathrm{a} i}^{2}$, is equal to 1 . In a recrystallized grain, the square of a certain $\eta_{\mathrm{b} i}$, i.e. $\eta_{\mathrm{b} i}^{2}$, is equal to 1 . On the grain boundary from the original grain to the recrystallized grain, the value of $\eta_{\mathrm{a} i}^{2}$ decreases gradually from 1 to 0 , and the value of $\eta_{\mathrm{b} i}^{2}$ increases gradually from 0 to 1. The energy of the grain boundary is higher than

Table 1 Parameters in the phase field simulation of the grains evolution for $17-4 \mathrm{PH}$ steel

\begin{tabular}{cccccccccccc}
\hline$L$ & $m$ & $n$ & $\alpha_{\mathrm{a}}$ & $\alpha_{\mathrm{b}}$ & $\beta$ & $\gamma$ & $\kappa_{\mathrm{a}}$ & $\kappa_{\mathrm{b}}$ & Grids & $\Delta t$ & $\Delta x$ \\
\hline 1 & 30 & 10 & 0.76 & 1 & 1 & 1 & 0.1 & 1 & $256 \times 256$ & 0.1 & 2 \\
\hline
\end{tabular}

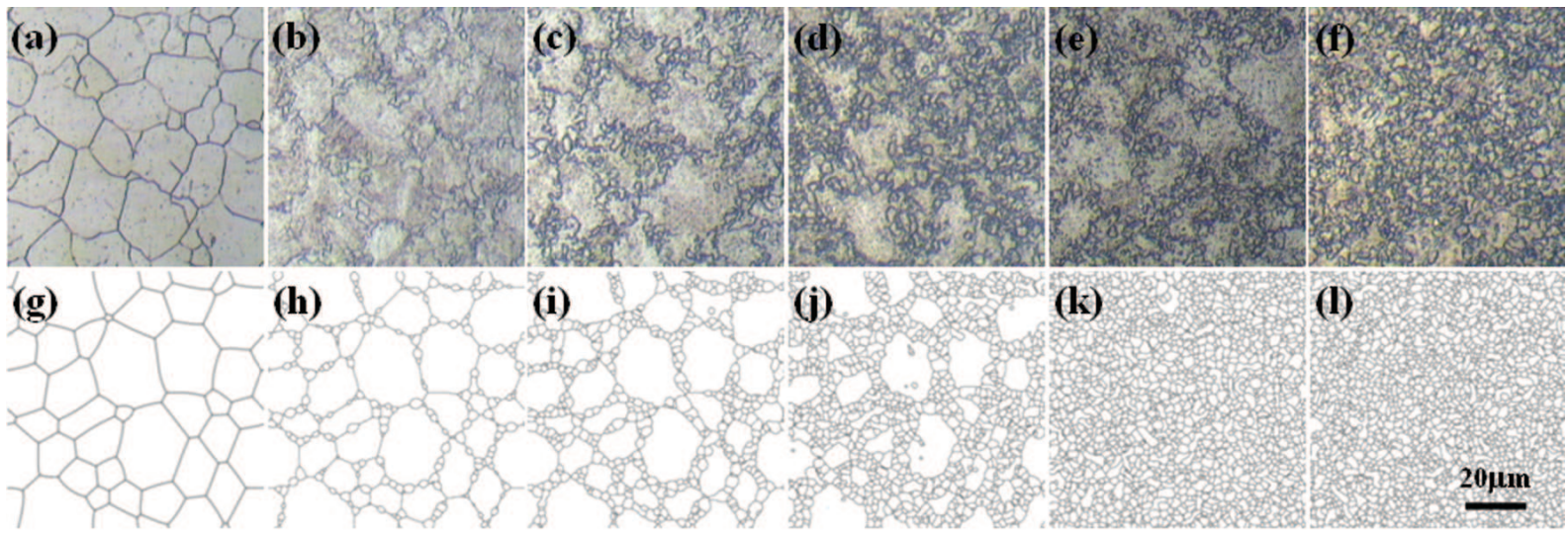

Fig. 2 Experimental and simulated grain morphologies of 17-4PH treated under different conditions: (a)(f) experimental results; (g)-(l) simulated results; (a) and (g) solution treatment (ST); (b) and (h) $\mathrm{ST}+$ a cyclic heat treatment $(\mathrm{CHT})$; (c) and (i) ST + two times CHT; (d) and (j) ST + three times CHT; (e) and (k) ST + four times CHT; (f) and (l) ST + five times CHT 
that inside the grain. The grain boundaries provide some energy for nucleation and reduce the nucleation work, so the formation of the recrystallized grains is easier on the grain boundary than inside the grains. With CHT proceeding, more and more recrystallized grains appear. Furthermore, as the amount of the recrystallized grains increase, these small grains extend gradually towards the original grain interior. After 5 times CHT in Fig. 2(f) and (l), almost all the grains become recrystallized grains, and the average grain size reaches the smallest. Obviously, the simulated grains agree well with the experimental ones. In addition, all the recrystallized grains present a similar size as shown in Fig. 2(b)-(f) and Fig. 2(h)-(l). The reason for this situation is that the recrystallized grains can grow just to the size within the treatment time (10 $\mathrm{min})$.

The experimental and simulated average grain size of the 17-4PH steel under different CHT is shown in Fig. 3. We can see that the average grain size after ST is about $13.2 \mu \mathrm{m}$, which can be decreased by $50 \%$ after a CHT. The average grain size decreases gradually and finally reaches a minimum around $2.2 \mu \mathrm{m}$ after 5 times CHT. This point can be demonstrated by our following simulations. The average grain size remains unchanged after 6 and 7 times CHT. This situation is because that the final average grain size of $2.2 \mu \mathrm{m}$ is just the recrystallized grain size in Fig. 2(b)-(f) and Fig. 2(h)-(l).

Fig. 4 shows the effects of phenomenological parameters of $\alpha_{\mathrm{a}}$ on the fraction of recrystallized region. The fraction of the recrystallized region decreases gradually with an increase in the value of parameter $\alpha_{\mathrm{a}}$ as seen from Fig. 4. Under $\alpha_{\mathrm{a}} \leq 0.734$ condition, the recrystallization takes place totally, i.e. both on the grain boundaries and inside the grains. And for $\alpha_{\mathrm{a}} \geq 0.781$, the recrystallization does not take place. As shown in Fig. 1, local energy density, as well as the total energy of the system, is determined by parameter $\alpha$. The decrease of the parameter $\alpha_{\mathrm{a}}$ corresponds to an increase in the energy of the original grains, which provide more energy for the nucleation of the recrystallized grains and make their formation

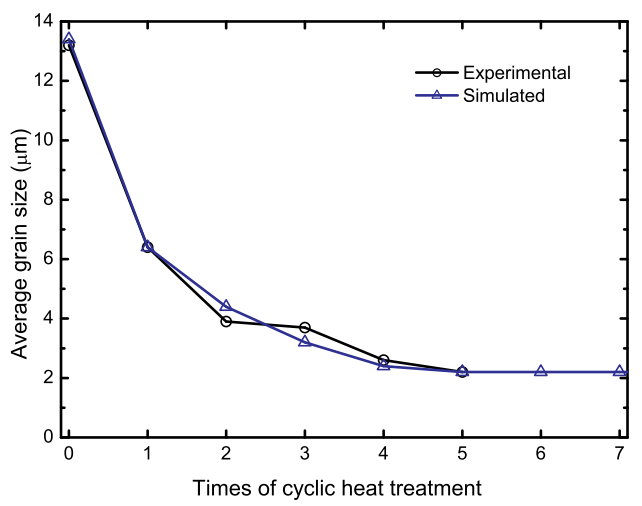

Fig. 3 Average grain size of $17-4 \mathrm{PH}$ steel treated under different times of CHT easier. Therefore, the fraction of the recrystallized region can be controlled by phenomenological parameter $\alpha$.

To discuss the effects of the gradient energy coefficient $\kappa_{\mathrm{b}}$ on the recrystallization, we perform the simulations for different $\kappa_{\mathrm{b}}$. The fraction of recrystallized region is assumed as $100 \%$ in these simulations in favor of the comparison of the results. Fig. 5 indicates that the average grain size increases with increasing the value of the gradient energy coefficient $\kappa_{\mathrm{b}}$. As mentioned in Ref. [20], the grain boundary energies are represented by gradient energy terms, and can be adjusted by varying the gradient energy coefficient. The larger the gradient energy coefficient, the higher the grain boundary energies. In order to decrease the total energy of the system, the amount of the grain boundary has to be decreased, which result in the increase of the grain size. Therefore, we could adjust the average grain size by varying the gradient energy coefficients $\kappa_{\mathrm{b}}$ to satisfy different systems. With different values of the phenomenological parameter $\alpha$ and gradient energy coefficients $\kappa_{\mathrm{b}}$, the present phase field can be extended to a variety of systems relative to recrystallization.

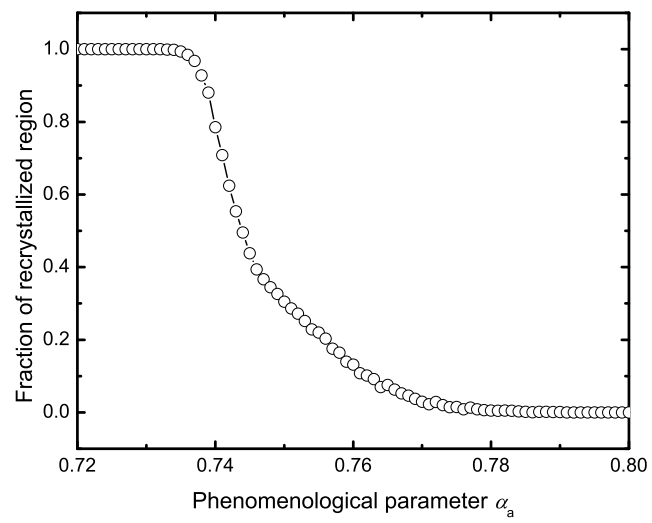

Fig. 4 Effects of phenomenological the parameters $\alpha_{\mathrm{a}}$ on the fraction of recrystallized region

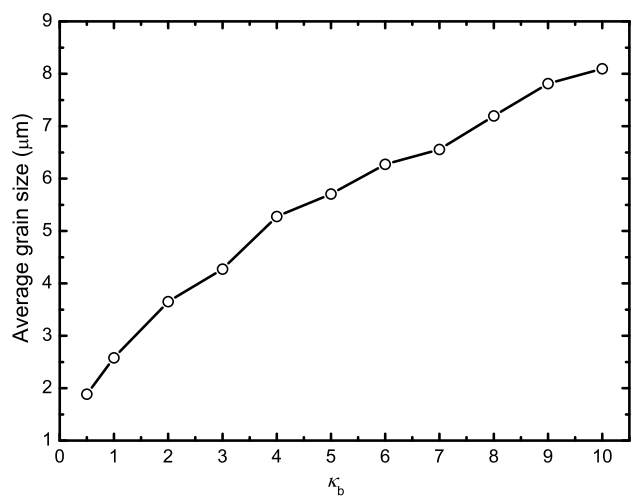

Fig. 5 Effects of gradient energy coefficients $\kappa_{\mathrm{b}}$ on the average grain size 


\section{Conclusions}

A phase field model is developed to simulate the grains' evolution of $17-4 \mathrm{PH}$ steel during cyclic heat treatment (CHT). Our simulation successfully reproduce the grains' morphologies of every CHT.

Both experimental and simulated results show that the average grain size of $13.2 \mu \mathrm{m}$ obtained by $1040{ }^{\circ} \mathrm{C} \times 1 \mathrm{~h}$ solution treatment for $17-4 \mathrm{PH}$ steel specimen can be refined to $2.2 \mu \mathrm{m}$ after 5 times CHT. The recrystallized grains appear mainly on the original grain boundaries.

In the phase field model, the fraction of the recrystallized region increases with reducing of phenomenological parameter $\alpha_{\mathrm{a}}$ in local energy function, and the average grain size increases with gradient energy coefficient $\kappa_{\mathrm{b}}$.

\section{Acknowledgements}

This work was financial supported by National Natural Science Foundation of China (No.51071061) and NSAF (No.11176011).

\section{REFERENCES}

[1] Y.S. Seo, Y.B. Chun and S.K. Hwang, Comput. Mater. Sci. 43 (2008) 512.

[2] B. Radhakrishnan, G. Sarma and T. Zacharia, Scr. Mater. 39 (1998) 1639.

[3] F. Caleyo, T. Baudin and R. Penelle, Scr. Mater. 46 (2002) 829.
[4] M. Kazeminezhad, Mater. Sci. Eng. A 496 (2008) 389.

[5] P. Mukhopadhyay, M. Loeck and G. Gottstein, Acta Mater. 55 (2007) 551.

[6] N. Xiao, C. Zheng, D. Li and Y. Li, Comput. Mater. Sci. 41 (2008) 366.

[7] M. Qian and Z.X. Guo, Mater. Sci. Eng. A 365 (2004) 180.

[8] R. Ding and Z.X. Guo, Acta Mater. 49 (2001) 3163.

[9] Y. Guo, Y. Wang and D. Zhao, Acta Mater. 55 (2007) 401.

[10] R.B. Godiksen, Z.T. Trautt, M. Upmanyu, J. Schi t z, D.J. Jensen and S. Schmidt, Acta Mater. 55 (2007) 6383.

[11] Z. Chen, C.L. Chen and L.M. Hao, Acta. Metall. Sin. (Engl. Lett.) 21 (2008) 444.

[12] Y. Zhang, C. Wang, D. Li and Y. Li, Acta. Metall. Sin. (Engl. Lett.) 22 (2009) 197.

[13] M.A. Zaeem, H. Yin and S.D. Felicelli, J. Mater. Sci. Technol. 28 (2012) 137.

[14] Y. Suwa, Y. Saito and H. Onodera, Mater. Sci. Eng. A 457 (2007) 132.

[15] Y. Suwa, Y. Saito and H. Onodera, Comput. Mater. Sci. 44 (2008) 286.

[16] M. Wang, B.Y. Zong and G. Wang, J. Mater. Sci. Technol. 24 (2008) 829.

[17] T. Takaki, Y. Hisakuni, T. Hirouchi, A. Yamanaka and Y. Tomita, Comput. Mater. Sci. 45 (2009) 881.

[18] J.W. Cahn and J.E. Hilliard, J. Chem. Phys. 28 (1958) 258.

[19] L.Q. Chen and W. Yang, Phys. Rev. B 50 (1994) 15752.

[20] L.Q. Chen, Scr. Metall. 32 (1995) 115.

[21] C.E. Krill III and L.Q. Chen, Acta Mater. 50 (2002) 3059 . 